Helgoländer wiss. Meeresunters. 31 444-456 1978)

\title{
Modalités de la contamination d'une chaine trophique marine benthique par l'argent $110 \mathrm{~m}$
}

\author{
3. Influence du mode de contamination sur l'organotropisme du \\ radionucléide"*
}

\author{
T.-C. AMIARD \\ Université de Nantes, Institut des Sciences de la Nature, Laboratoire de Biologie Marine; \\ 2 Rue de la Houssinière, F-44072 Nantes Cedex, France
}

\begin{abstract}
Ag}$ contamination mechanisms in a marine benthic food chain. 3. Influence of the mode of contamination upon the distribution of the radionuclide. The distribution

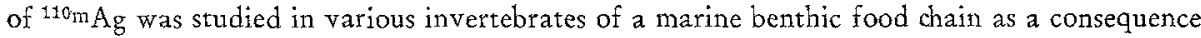
of the immersion in contaminated seawater and/or ingestion of radioactive food. The studies on ${ }^{110 \mathrm{~m} A g}$ distribution were carried out at the end of the experimental contamination (when equilibrium was reached) and during the slow stage of elimination. After contamination via the surrounding water, uptake of $110 \mathrm{mAg}$ occurs mainly in external organs: shells (34\%) and mantle $(16 \%)$ of the bivalve Scrobicularia plana, exoskeleton $(40 \%)$ and gills $(38 \%)$ of the crab Carcinus maenas. On the contrary, contamination via food is responsible for a considerable accumulation of $110 \mathrm{mAg}$ in the digestive glands of $S$. plana $(75 \%)$ and C. maenas $(56 \%)$. Moulting plays an important role in the decontamination of crabs following immersion in radioactive seawater owing to the strong contamination of the exuviae. The elimination of $110 \mathrm{mAg}$ from the different organs of the animals examined depends upon the mode of contamination: it is generally fast in external organs and slow in the digestive gland. Radiation doses for molluscs and crustaceans were calculated following experimental contaminations by water and food. A significant contamination of external organs is responsible for a radiation dose (in the centre of the test animals) weaker than that instigated by the accumulation of ${ }^{110} \mathrm{~m} \mathrm{Ag}$ in the digestive gland. Thus, with regard to contamination, the ambient water is important; but, from the point of view of irradiation, radioactive food is generally more important.
\end{abstract}

\section{INTRODUCTION}

Nous avons vu précédemment l'importance de l'argent $110 \mathrm{~m}$ dans la contamination des organismes marins par l'intermédiaire de l'eau (Pouvreau \& Amiard, 1974; Amiard, 1978a) ou par l'intermédiaire de la nourriture (Amiard, 1978b).

La présence des isotopes $110 \mathrm{~m}$ et $108 \mathrm{~m}$ de l'argent dans les retombées des explosions nucléaires et celle de l'isotope $110 \mathrm{~m}$ dans les rejets liquides des centrales

* Cet article a pu être rédigé à l'occasion d'études effectuées dans les laboratoires de la Section de Radioécologie du Commissariat à l'Energie Atomique (DPr/SERE). 
électronucléaires et des usines de traitement des combustibles irradiés a été signalée par plusieurs auteurs (Seymour, 1963; Folsom \& Young, 1965; Preston et al., 1968; Folsom et al., 1970; Beasley \& Held, 1971; Grismore et al., 1972; Fukai \& Murray, 1974; Scheidhauer et al., 1974).

Un paramètre important de la radiotoxicité de l'argent $110 \mathrm{~m}$ vis à vis de la flore et de la faune sera sa distribution dans les organismes marins. En effet, la dose d'irradiation absorbée par l'organisme dépend principalement de la répartition interne ou externe du radionucléide. De plus, son organotropisme dans les espèces comestibles conditionnera son transfert à l'Homme par voie alimentaire.

Aussi, nous allons passer en revue l'influence du mode de contamination (via l'eau ou via la nourriture) sur l'organotropisme de l'argent $110 \mathrm{~m}$ chez Scrobicularia plana et Carcinus maenas respectivement consommateur I et II d'une chaîne trophique marine benthique naturelle (Amiard, 1978a, b).

Ces données nous permettrons d'estimer les doses d'irradiation dues aux photons subies par les animaux en fonction de la voie de contamination. Nous comparerons les doses d'irradiation subies par les organes critiques internes avec celles subies par l'animal supposé contaminé uniformément ou celles dues aux organes critiques externes.

\section{PROTOCOLE EXPERIMENTAL}

\section{Contaminations par l'eau}

Les conditions expérimentales ont été identiques pour les deux espèces étudiées. Les animaux, placés dans des seaux contenant une eau de mer aérée en permanence, étaient maintenus à la température de $17 \pm 1^{\circ} \mathrm{C}$. Les contaminations ont été effectuées à partir d'une source d'argent $110 \mathrm{~m}$ sous forme de nitrate d'argent en solution nitrique $(2,7 \mathrm{~N})$, d'activité spécifique $7,8 \mathrm{Ci} / \mathrm{g}$, à raison de 1 ou $5 \mu \mathrm{Ci} / 1$ pour les Scrobiculaires et $4 \mu \mathrm{Ci} / 1$ pour les Crabes.

Lorsque l'état d'équilibre était atteint (c'est à dire après 22 à 44 jours pour les Scrobiculaires et après 30 jours pour les Crabes) et au cours de la phase longue de la décontamination (après 30 jours pour les Scrobiculaires, 10 à 21 jours pour les Crabes), les individus étaient prélevés, rincés et disséqués.

\section{Contaminations par la nourriture}

La nourriture des Scrobiculaires était constituée de Navicules (Navicula incerta ou $N$. biskanteri) qui leur étaient fournies sous forme de cultures axéniques. Les Navicules, cultivées dans un milieu contaminé à raison de $5 \mu \mathrm{Ci} / 1$ d'argent $110 \mathrm{~m}$, avaient accumulé des quantités importantes de ce radionucléide ( 5 à $40 \mu \mathrm{Ci} / \mathrm{g}$ frais).

Les Crabes recevaient deux repas hebdomadaires composés d'une Scrobiculaire elle-même contaminée uniquement à partir de Navicules radioactives.

Les dissections et les mesures de radioactivité des deux espèces ont été effectuées à la fin de la phase de contamination soit 12 à 29 repas pour les Scrobiculaires et 
7 à 12 repas pour les Crabes. Les Scrobiculaires avaient pratiquement atteint l'état d'équilibre tandis que les Crabes en étaient encore loin. Une autre série de dissection a été effectuée au cours de la phase longue de la décontamination (soit 10 jours pour les Scrobiculaires et 72 jours pour les Crabes).

\section{Contaminations simultanées par l'eau et la nouriture}

Des Scrobiculaires et des Crabes étaient contaminés à la fois par l'eau de mer et par leur nourriture (Diatomées pour les Scrobiculaires, Scrobiculaires pour les Crabes).

Une première série de dissections a été effectuée à la fin de la phase de contamination c'est à dire après 34 à 56 repas pour les Scrobiculaires et 8 repas pour les Crabes. Une deuxième série de dissections a été réalisée durant la phase longue de la décontamination des Scrobiculaires soit après 41 à 90 jours de décontamination.

\section{Dissection et techniques de comptage de la radioactivité}

Nous prélevions sept organes et groupes d'organes différents chez Scrobicularia plana: coquille, siphons, hépatopancréas, pied, restes de la masse viscérale, manteau et muscles adducteurs.

Chez le Crabe, nous prélevions huit organes ou groupes d'organes: hémolymphe, pièces buccales, carapace, moulinet gastrique, branchies, hépatopancréas, organes génitaux et muscles. Par ailleurs, un certain nombre d'autres organes étaient isolés de façon à connaître la radioactivité totale contenue dans sept compartiments: exosquelette (avec une petite fraction de muscles), branchies, tube digestif, hépatopancréas, organes génitaux, muscles (avec endosquelette) et hémolymphe.

La radioactivité de chaque échantillon était mesurée sur un cristal plat d'iodure de sodium activé au thallium associé soit à un sélecteur monocanal, soit à un sélecteur multicanaux. Les résultats étaient exprimés soit en $\mathrm{nCi} / g$ de poids frais, soit en $\mathrm{nCi}$ pour l'organe in toto en tenant compte du rendement des appareils de détection, de la géométrie des échantillons, de la décroissance radioactive du radionucléìde et en déduisant la radioactivité ambiante.

$$
\text { Expression des résultats }
$$

La somme des radioactivités par unité de poids ( $\mathrm{nCi} / \mathrm{g}$ frais) des sept organes des Scrobiculaires ou des huit organes des Crabes était assimilée à $100 \%$ et nous en déduisions le pourcentage correspondant pour chacun des organes.

De plus, dans le cas des Crabes nous additionnions les radioactivités totales 
(nCi) des sept compartiments, l'assimilions à $100 \%$ et en déduisions le pourcentage correspondant pour chacun des compartiments.

Nous calculions alors les moyennes, les écarts-types à la moyenne (ou erreur standard) et les intervalles de confiance (ou coefficient de sécurité) à $95 \%(2 \mathrm{sm}$ ) pour chacun des organes (Lamotte, 1967; Lefort, 1967).

Nous effectuions alors une comparaison deux à deux des moyennes des divers groupes d'animaux à l'aide du test $t$ de Student-Fisher et notions si les moyennes étaient significativement différentes avec $95 \%$ de confiance (to,05) ou $99 \%$ de confiance $(\mathrm{t} 0,01)$.

Estimation de la dose d'irradiation interne subie par les animaux d'expérience

Pour chaque espèce, nous avons cherché à déterminer les doses d'irradiation interne dues aux photons ( $\gamma$ et $\mathrm{X}$ ) et aux rayonnements $\beta$ subies par les organismes in toto en un point central après contamination par l'eau ou la nourriture. Nous avons cherché à estimer également les doses d'irradiation dues aux photons et aux rayonnements $\beta$ résultant de la contamination des organes critiques pour les deux voies de contamination (eau et nourriture). Nous n'avons pas tenu compte de l'irradiation due à la contamination du milieu externe.

Nous avons sélectionné les caractéristiques physiques des photons et des rayonnements $\beta$ (énergie, fréquence) dans Lederer et al. (1967) et Legrand et al. (1975) et les coefficients d'atténuation et d'absorption dans Joffre \& 2 Pages (1968).

Pour le calcul de la dose d'irradiation due aux photons, les organismes in toto et l'hépatopancréas ont été assimilés à des cylindres (volume) tandis que les organes (coquille, ... o) ont été assimilés à la paroi latérale et aux deux disques d'un cylindre (surface). Les formules mathématiques nous ont été fournies par Rockwell (1956) et par Fitzgerald et al. (1967). Pour les rayons $\beta$ nous avons supposé l'organisme ou l'organe critique comme un milieu infini puis nous avons appliqué un facteur de correction tenant compte des dimensions réelles de l'organisme et du parcours maximal des électrons dans les tissus mous (Fitzgerald et al., 1967). L'ensemble de ces calculs a été détaillé dans Amiard (1978c).

\section{RESULTATS}

\section{Résultats concernant Scrobicularia plana}

Les Figures $1 \mathrm{~A}_{1}, 1 \mathrm{~B}_{1}$ et $1 \mathrm{C}_{1}$ permettent de comparer la répartition de l'argent $110 \mathrm{~m}$ selon la voie de contamination. La part de la coquille (to,01), du manteau (to,01) et des siphons $(\mathbf{t} 0,05)$ est nettement plus élevée quand la contamination se fait par l'eau seule que lorsque le vecteur nourriture est intervenu. Le phénomène est inverse pour l'hépatopancréas ( $t 0,01)$ et la masse viscérale $(t 0,01)$. Il en est sensiblement 
de même lorsque l'on compare la contamination simultanée par l'eau et la nourriture avec la contamination par la nourriture seule. Dans ce cas, l'ingestion de nourriture contaminée entraîne une contamination prépondérante pour l'hépatopancréas (to,01) et la masse viscérale (to,05) et la contamination simultanée par l'eau et la nourriture provoque une accumulation de l'argent $110 \mathrm{~m}$ principalement dans la coquille (to,01) et les siphons ( $\mathrm{t}_{0,05}$ ) (Fig. $1 \mathrm{~B}, \mathrm{C}_{1}$ ). Par contre, nous ne distinguons aucune différence significative $(t 0,01)$ ou $(t 0,05)$ entre la contamination due à l'eau et la contamination due simultanément à l'eau et à la nourriture (Fig. 1 A1, $\mathrm{B}_{1}$ ).
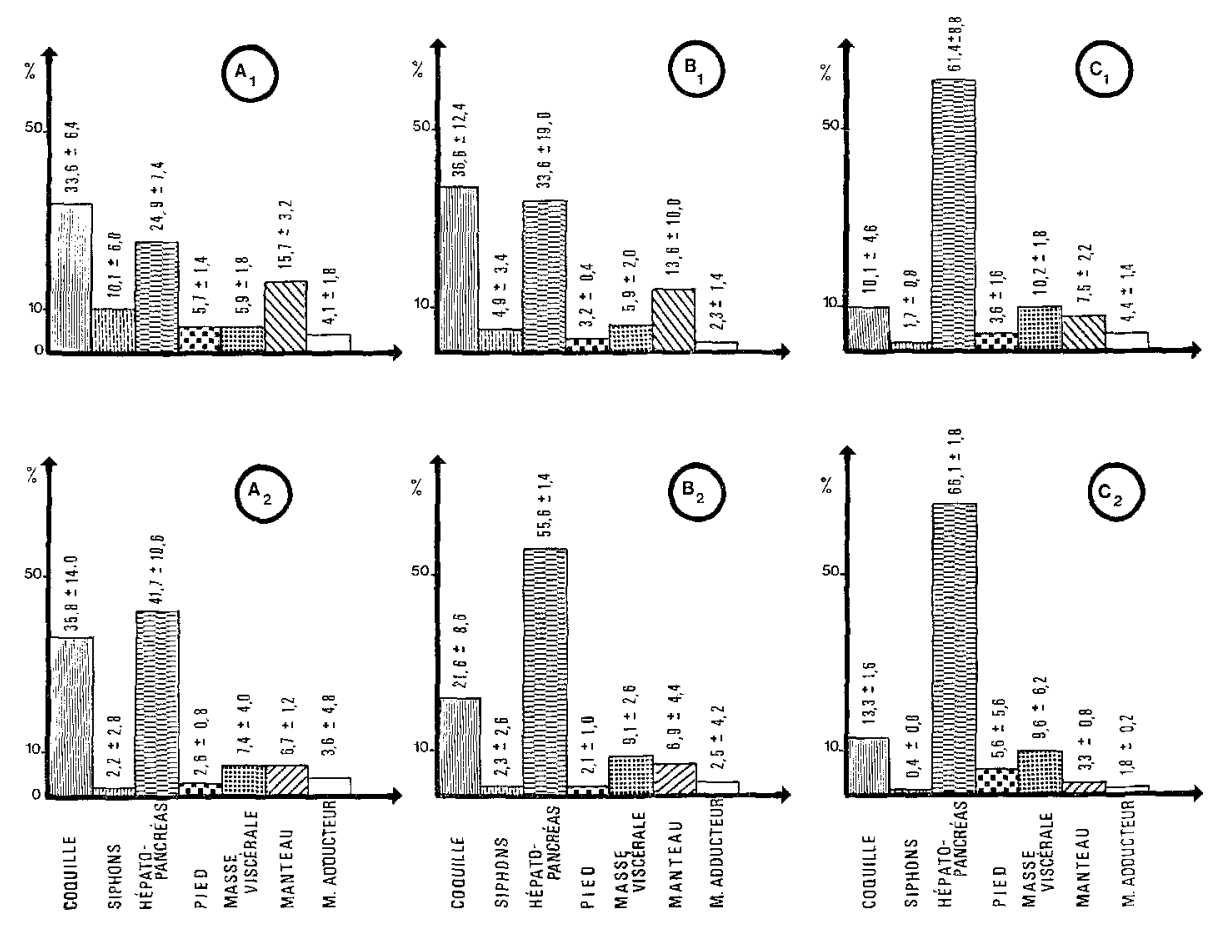

Fig. 1: Répartition de l'argent $110 \mathrm{~m}$ dans l'organisme de Scrobicularia plana en fonction du mode de contamination ( $A_{1}$ eau; $B_{1}$ eau et nourriture; $C_{1}$ nourriture) et au cours de la décontamination après contamination par l'eau $\left(\mathrm{A}_{2}\right)$, l'eau et la nourriture $\left(\mathrm{B}_{2}\right)$ et la nourriture $\left(\mathrm{C}_{2}\right)$. (Résultats exprimés en pourcentages de la somme des radioactivités par unité de poids de tous les organes - Intervalle de confiance à $95 \%$ )

Les Figures $1 \mathrm{~A} 2,1 \mathrm{~B}_{2}$ et $1 \mathrm{C}_{2}$ indiquent la distribution de l'argent $110 \mathrm{~m}$ chez la Scrobiculaire au cours de la phase longue de l'élimination. Cette répartition ne présente pas de différence significative entre les individus ayant été contaminés par l'eau et ceux contaminés par l'eau et la nourriture (Fig. 1 A2, B2), ni entre ces derniers et ceux contaminés par la nourriture seule (Fig. 1 B2, C2). Par contre, les animaux contaminés par l'eau ont leur manteau $(t 0,01)$ plus riche et leur hépatopancréas $(\mathrm{t} 0,05)$ moins riche en argent $110 \mathrm{~m}$ que les Mollusques contaminés par la nourriture (Fig. $1 \mathrm{~A}_{2}, \mathrm{C}_{2}$ ). 
Après contamination par l'eau, le manteau $\left(t_{0,05}\right)$ des Scrobiculaires se décontamine plus rapidement que les autres organes (Fig. $1 \mathrm{~A} 1, \mathrm{~A} 2$ ). Par contre, nous ne trouvons aucune différence significative dans la décontamination des Scrobiculaires après contamination soit par l'eau et la nourriture, soit par la nourriture seule (Fig. 1 B1-B2, $\mathrm{C}_{1-\mathrm{C}_{2}}$ ).

\section{Résultats concernant Carcinus maenas}

La Figure 2 permet de comparer la répartition relative de l'argent $110 \mathrm{~m}$ chez le Crabe sur la base de l'unité de poids. L'argent $110 \mathrm{~m}$ se répartit préférentiellement dans les pièces buccales $(t 0,01)$ et les branchies ( $t 0,01)$ après contamination par l'eau
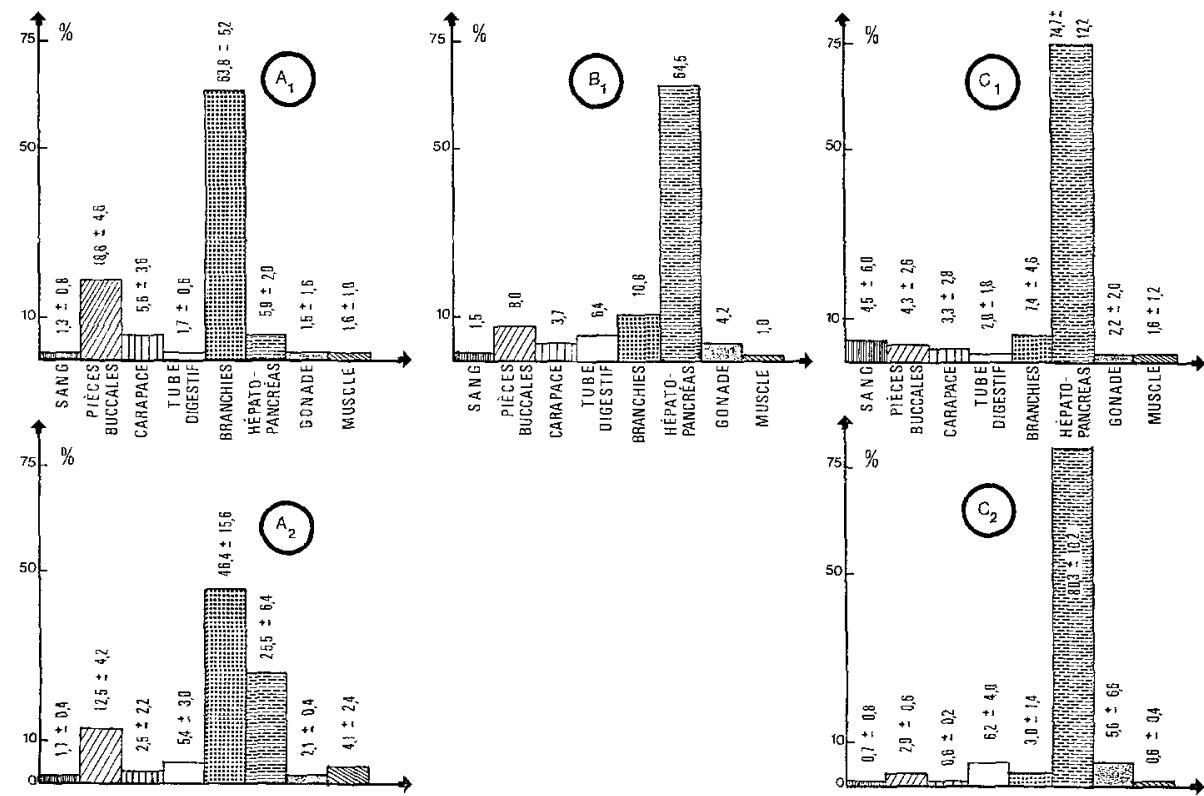

Fig. 2: Répartition de l'argent $110 \mathrm{~m}$ dans l'organisme de Carcinus maenas en fonction du mode de contamination ( $A_{1}$ eau; $B_{1}$ eau et nourriture; $C_{1}$ nourriture) et au cours de la décontamination après contamination par l'eau $\left(\mathrm{A}_{2}\right)$ et la nourriture $\left(\mathrm{C}_{2}\right)$. (Résultats exprimés en pourcentages de la somme des radioactivités par unité de poids de tous les organes Intervalle de confiance à $95 \%$ )

et dans l'hépatopancréas (to,01) après contamination par l'intermédiaire de la nourriture (Fig. 2 A1-C1). Dans la Figure 3, les divers organes sont représentés en fonction de leur radioactivité globale. Dans ce cas également, l'argent se retrouve surtout dans les organes externes, branchies $(t 0,01)$ et exosquelette ( $t 0,01)$, après pollution par l'eau et dans l'hépatopancréas (to,0r), le tube digestif (to,01) et l'hémolymphe $(\mathrm{t} n, 05)$ après contamination par la nourriture (Fig. 3 A1-C1). 
En raison de l'effectif trop restreint du groupe contaminé par l'eau et la nourriture ( 1 seul individu) il n'est pas possible d'effectuer la comparaison à l'aide du test $t$ de Student-Fisher. Toutefois, l'observation des Figures 2 et 3 nous indique une grande similitude dans la répartition de l'argent $110 \mathrm{~m}$ entre les groupes $\mathrm{B}_{1}$ et $\mathrm{C} 1$ c'est à dire entre le groupe contaminé par l'eau et la nourriture et le groupe contaminé par la nourriture seule (Fig. $2 \mathrm{~B}_{1}-\mathrm{C}_{1}$ et $3 \mathrm{~B} 1-\mathrm{C} 1$ ).
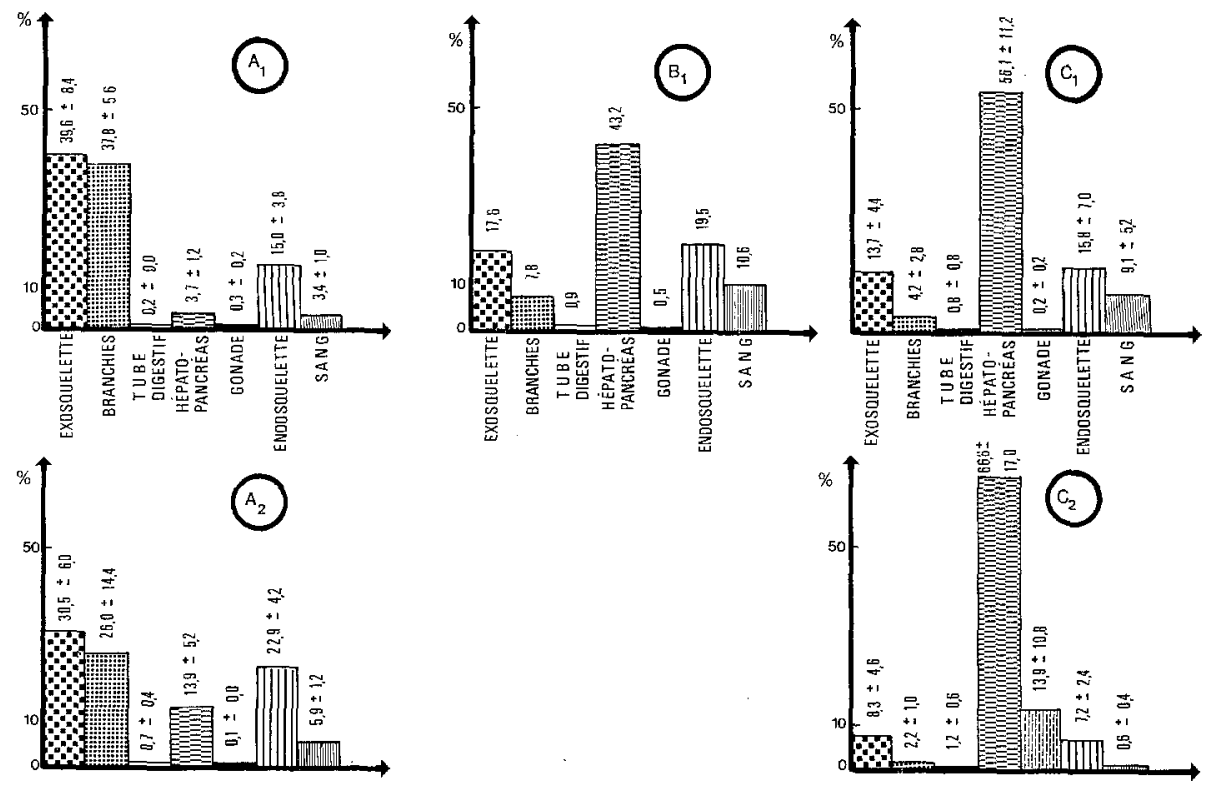

Fig. 3: Répartition de l'argent $110 \mathrm{~m}$ dans l'organisme de Carcinus maenas en fonction du mode de contamination ( $A_{1}$ eau; $B_{1}$ eau et nourriture; $C_{1}$ nourriture) et au cours de la décontamination après contamination par l'eau $\left(\mathrm{A}_{2}\right)$ et la nourriture $\left(\mathrm{C}_{2}\right)$. (Résultats exprimés en pourcentages de la radioactivité totale de l'organisme - Intervalle de confiance à $95 \%$ )

La répartition de l'argent $110 \mathrm{~m}$ chez le Crabe dans la phase longue de la décontamination indique une opposition quasi-identique entre les Crustacés contaminés par l'eau et ceux contaminés par la nourriture. Ainsi, que l'on compare la distribution par unité de poids $(\mathrm{nCi} / \mathrm{g}$ ) ou pour l'organe entier (nCi), les organes externes (branchies, carapace et exosquelette) et les muscles accumulent significativement plus (to,01) et l'hépatopancréas significativement moins (to,01) après contamination par l'eau qu'après contamination par la nourriture (Fig. $2 \mathrm{~A}_{2}-\mathrm{C}_{2}$ et $3 \mathrm{~A} 2-\mathrm{C} 2$ ).

Après contamination par l'eau, les branchies se décontaminent plus rapidement et l'hépatopancréas, le tube digestif et l'hémolymphe se décontaminent moins vite que les autres organes. Dans le cas de la contamination par la nourriture, l'exosquelette, les muscles et l'hémolymphe se décontaminent plus rapidement que les autres organes (Fig. 2 A1-A2 et $\mathrm{C}_{1-\mathrm{C}_{2}}$ et Fig. 3 A1- $\mathrm{A}_{2}$ et $\mathrm{C}_{1-\mathrm{C}_{2}}$ ). 
Influence de la mue sur l'organotropisme de l'argent $110 \mathrm{~m}$ chez Carcinus maenas

Nous avons vu qu'après contamination par l'eau, le Crustacé C. maenas pouvait perdre jusqu'à $78,9 \%$ de sa radioactivité totale dans sa mue et $92,3 \%$ de sa radioactivité par unité de poids (Amiard, 1978a).

La distribution de l'argent $110 \mathrm{~m}$ dans l'exuvie de C. maenas se fait principalement dans les branchies $(46,6 \%)$ et les pièces buccales $(17,9 \%)$ alors que ces deux groupes d'organes ne représentent pondéralement que respectivement $12,5 \%$ et $15,9 \%$ du poids de l'exuvic. Globalement la radioactivité est surtout présente dans les branchies $(33,3 \%)$ et les pattes $\mathrm{P}_{2}$ à $\mathrm{P}_{5}(31,8 \%)$.

Nous avons constaté qu'un Crabe après la mue se recontamine directement par l'eau plus lentement que les animaux parvenus au stade C4 (Drach, 1939; Drach \& Tchernigovtzeff, 1967). La répartition de l'argent $110 \mathrm{~m}$ après treize jours de contamination est différente de celle observée après une contamination durant laquelle les animaux n'ont pas mué. Nous n'avons pu effectuer de test $t$ en raison du faible effectif de cette catégorie (1 seul individu). Nous observons cependant une nette diminution de l'importance des organes externes, en particulier des branchies, et au contraire une accumulation préférentielle de l'argent $110 \mathrm{~m}$ dans les organes internes: tube digestif, hépatopancréas, hémolymphe et organes génitaux. Parmi les organes externes, la carapace est l'organe qui se recontamine le plus rapidement et après treize jours de contact avec l'eau radioactive retrouve sa même importance qu'avant la mue et même sensiblement plus.

Doses d'irradiation internes subies par les animaux

D'après nos données expérimentales, les Scrobiculaires contaminées par l'eau subissent globalement une dose d'irradiation par les photons onze fois plus importante que celles contaminées par voie alimentaire. Par contre, les organes critiques à la suite d'une contamination directe par l'eau (coquille et manteau) sont responsables d'une dose d'irradiation au centre de l'animal vingt trois fois plus faible que la dose d'irradiation subie par l'organe de stockage principal (hépatopancréas) après contamination par la nourriture (Tabl. 1). L'hépatopancréas, après contamination par voie alimentaire, est quarante sept fois plus irradié que l'animal contaminé uniformément.

Des résultats comparables sont observés pour les doses d'irradiation subies par les Crabes avec des différences encore plus significatives (Tabl. 1). Les Crabes supposés contaminés uniformément par l'eau sont cent quatorze fois plus irradiés que ceux contaminés uniformément par la nourriture. L'hépatopancréas, après contamination par voie alimentaire, est soixante douze fois plus irradié que l'animal contaminé uniformément. Par contre, les organes critiques externes après contamination par l'eau ont une influence assez faible sur l'irradiation des Crabes au point central (Tabl. 1). 


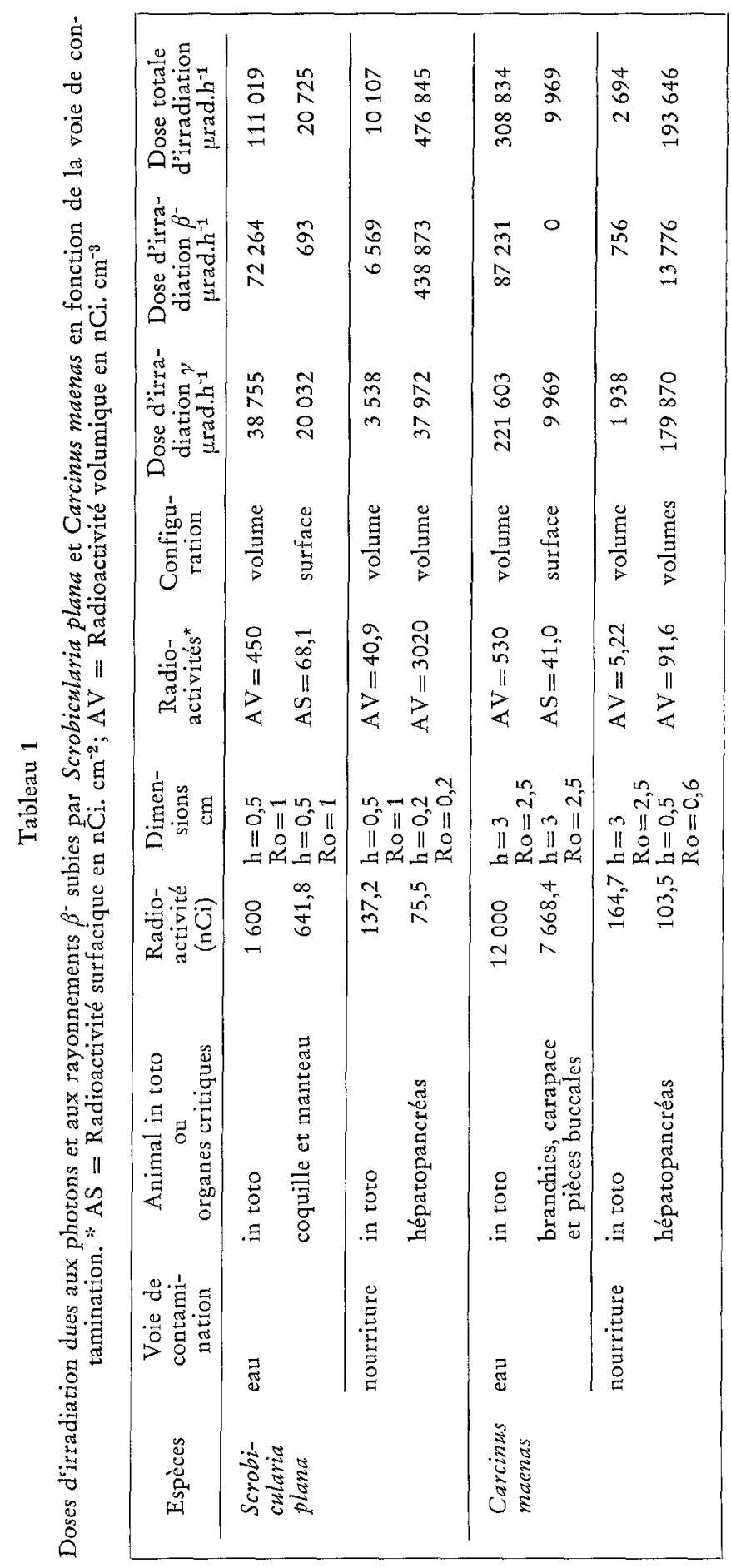




\section{Tableau 2}

Données bibliographiques sur l'organotropisme de l'argent $110 \mathrm{~m}$ et de l'argent stable chez quelques Mollusques et Crustacés marins. a: Pouvreau \& Amiard (1974), b: Greig (1975), c: Amiard (1978c), d: Isakson \& Seymour (1968), e: Zesenko (1965), f: Lowman et al. (1967)

\begin{tabular}{|c|c|c|c|}
\hline Espèces & Organes & $\begin{array}{l}\text { F.C. } \\
110 \mathrm{mAg}\end{array}$ & Teneur en argent stable \\
\hline \multicolumn{4}{|l|}{ Mollusques } \\
\hline \multirow{2}{*}{ Mytilus edulis } & chair & $500 \div a$ & \\
\hline & coquille & $250 \mathrm{a}$ & \\
\hline \multirow{2}{*}{ Patella oulgata } & chair & 1100 a & \\
\hline & $\begin{array}{l}\text { viscères } \\
\text { coquille }\end{array}$ & $\begin{array}{r}4400^{-5} \mathrm{a} \\
50 \mathrm{a}\end{array}$ & \\
\hline \multirow[t]{2}{*}{ Ostrea edulis } & chair & $32000 * a$ & \\
\hline & coquille & $50 \mathrm{a}$ & \\
\hline Cardium edule & & & \\
\hline \multirow{2}{*}{ Haliotis tuberculata } & $\begin{array}{l}\text { coquille } \\
\text { chair }\end{array}$ & 3000 a & \\
\hline & $\begin{array}{l}\text { coquille } \\
\text { hépatopancréas }\end{array}$ & $\begin{array}{rl}60 & a \\
12000 & a\end{array}$ & \\
\hline Busycon canaliculatum & $\begin{array}{l}\text { p. molles } \\
\text { glande dig. } \\
\text { rein } \\
\text { branchies }\end{array}$ & & $\begin{array}{clll}7,9 & \text { à } & 8,2 & \mathrm{~b} \mu \mathrm{g} / \mathrm{g} \mathrm{fr} . \\
23 & \grave{a} 32 & \mathrm{~b} \mu \mathrm{g} / \mathrm{g} \text { fr. } \\
1,3 & \text { à } & 2,5 & \mathrm{~b} \mu \mathrm{g} / \mathrm{g} \text { fr. } \\
0,48 & \text { à } & 0,53 & \mathrm{~b} \mu \mathrm{g} / \mathrm{g} \text { fr. }\end{array}$ \\
\hline \multicolumn{4}{|l|}{ Crustacés } \\
\hline \multirow[t]{3}{*}{ Carcinus maenas } & $\begin{array}{l}\text { hémolymphe } \\
\text { hépatopancréas }\end{array}$ & & $\begin{array}{c}\mathrm{ND} c \\
0,38 \pm 0,52 \mathrm{c} \mu \mathrm{g} / \mathrm{g} \mathrm{sec}\end{array}$ \\
\hline & carapace & $150 \mathrm{e}$ & \\
\hline & $\begin{array}{l}\text { coeur, gon., muscle } \\
\text { foie }\end{array}$ & $\begin{array}{r}10-20 \mathrm{e} \\
80 \mathrm{e}\end{array}$ & \\
\hline \multirow[t]{2}{*}{ Cancer pagurus } & branchie & $125 \mathrm{a}$ & \\
\hline & carapace & 180 a & \\
\hline Cancer irroratus & $\begin{array}{l}\text { muscle } \\
\text { glande dig. }\end{array}$ & & $\begin{array}{l}0,21 \text { à } 0,78 \text { b } \mu g / g \text { fr. } \\
1,1 \text { à } 6,3 \quad \text { b } \mu g / g \text { fr. }\end{array}$ \\
\hline \multirow[t]{2}{*}{ Litbodes sp. } & muscle & & $0,0024 \mathrm{~d} \mu \mathrm{g} / \mathrm{g} \mathrm{sec}$ \\
\hline & hépatopancréas & & $0,023 \mathrm{~d} \mu \mathrm{g} / \mathrm{g} \mathrm{sec}$ \\
\hline \multirow[t]{7}{*}{ Panulinus argus } & exosquelette & $8,8 \mathrm{f}$ & $0,1000 \mathrm{f} \mu g / g$ frais \\
\hline & tube digestif & $429,0 \mathrm{f}$ & $0,1597 \mathrm{f} \mu \mathrm{g} /$ gfrais \\
\hline & branchies & $9,6 \mathrm{f}$ & $0,0036 \mathrm{f} \mu \mathrm{g} / \mathrm{g}$ frais \\
\hline & gonades & $32,5 \mathrm{f}$ & $0,0121 \mathrm{f} \mu \mathrm{g} / \mathrm{g}$ frais \\
\hline & cerveau & $429,0 \mathrm{f}$ & $0,0089 \mathrm{f} \mu \mathrm{g} / \mathrm{gfrais}$ \\
\hline & hépatopancréas & $6041,0 \mathrm{f}$ & $2,242 \mathrm{f} \mu \mathrm{g} / \mathrm{g}$ frais \\
\hline & muscle & $12,6 \mathrm{f}$ & $0,0047 \mathrm{f} \mu \mathrm{g} / \mathrm{gfrais}$ \\
\hline \multirow{6}{*}{ Panulirus argus*: } & exosquelette & $2,6 \mathrm{f}$ & $0,0010 \mathrm{f} \mu \mathrm{g} / \mathrm{gfrais}$ \\
\hline & tube digestif & $11,8 \mathrm{f}$ & $0,0044 \mathrm{f} \mu \mathrm{g} / \mathrm{gfrais}$ \\
\hline & branchies & $5,1 \mathrm{f}$ & $0,0018 \mathrm{f} \mu \mathrm{g} / \mathrm{g}$ frais \\
\hline & gonades & $1,0 \mathrm{f}$ & $0,0004 \mathrm{f} \mu \mathrm{g} / \mathrm{gfrais}$ \\
\hline & hépatopancréas & $4990,0 \mathrm{f}$ & 1,855 f $\mu g / g$ grais \\
\hline & muscle & $0,9 \mathrm{f}$ & $0,0003 \mathrm{f} \mu \mathrm{g} / \mathrm{g}$ frais \\
\hline \multicolumn{4}{|c|}{$\begin{array}{l}\text { * Etat d'équilibre non atteint } \\
\text { * après } 48 \text { h de décontamination } \\
\text { ND: non détecté }\end{array}$} \\
\hline
\end{tabular}




\section{DISCUSSION ET CONCLUSIONS}

Nous avons vu que la répartition de l'argent $110 \mathrm{~m}$ chez la Scrobiculaire se faisait surtout dans la coquille, l'hépatopancréas et le manteau après contamination par l'eau et principalement dans l'hépatopancréas après contamination par la nourriture. Chez le Crabe, après contamination par l'eau l'argent $110 \mathrm{~m}$ se retrouve surtout dans les branchies et les pièces buccales tandis que l'hépatopancréas retient la quasi-totalité de l'argent dans le cas de contamination par la nourriture.

Quel que soit le mode de contamination et l'espèce, l'hépatopancréas est l'organe stockeur par excellence à l'exception des Crabes contaminés par l'eau. Dans ce dernier cas la quantité d'argent $110 \mathrm{~m}$ accumulée sur les organes externes semble être due à un phénomène passif d'adsorption. En effet, pour l'exuvie nous avons pu observé que les pattes $\mathrm{P}_{2}$ à $\mathrm{P}_{5}$ concentrent plus (15,8\% sur la base de l'unité de poids et $31,5 \%$ par rapport à la radioactivité totale) d'argent $110 \mathrm{~m}$ que les pinces $\mathrm{P}_{1}$ (respectivement $6,2 \%$ et 7,8\%). Or, la différence de poids est beaucoup moins forte: $34,9 \%$ pour les pattes et $22,2 \%$ pour les pinces. Par contre, les pattes représentent plus de surface par rapport au poids que les pinces et leur pouvoir de piégeage est accentué par la présence de nombreuses soies.

L'importance de l'hépatopancréas dans le stockage de l'argent se vérifie également dans les quelques mesures d'argent $110 \mathrm{~m}$ ou d'argent $108 \mathrm{~m}$ effectuées in situ. Ces deux isotopes ne sont détectés en grande quantité que dans le foie ou l'hépatopancréas des Céphalopodes, Crustacés et Téléostéens (Folsom \& Young, 1965; Folsom et al., 1970; Beasley \& Held, 1971). Il en est de même pour l'argent stable (Tabl. 2) (Isackson \& Seymour, 1968; Greig, 1975; Amiard, 1978c).

Les rares expériences de répartition de l'argent $110 \mathrm{~m}$ chez les Mollusques et les Crustacés aboutissent aux mêmes conclusions à quelques exceptions près (Tabl. 2) (Zesenko, 1965; Lowman et al., 1967; Pouvreau \& Amiard, 1974).

Au cours de l'élimination, la répartition de l'argent $110 \mathrm{~m}$ chez la Scrobiculaire varie assez peu sauf pour le manteau après une contamination par l'eau. Par contre, pour les Crabes après contamination par l'eau ce sont surtout les branchies qui se décontaminent vite tandis que les organes internes comme l'hépatopancréas, l'hémolymphe et le tube digestif se décontaminent lentement. Après contamination par la nourriture, certains organes internes comme les muscles et l'hémolymphe, et organe externe comme la carapace se décontaminent rapidement.

Après une contamination expérimentale de Panulirus argus par l'argent $110 \mathrm{~m}$ les chercheurs du Puerto Rico Nuclear Center constatent comme nous-même une grande rétention de l'argent $110 \mathrm{~m}$ et de l'argent stable dans l'hépatopancréas et par contre une élimination rapide dans les muscles, les gonades et le cerveau (Lowman et al., 1967).

Ainsi l'organotropisme d'un radionucléide chez un organisme marin va être conditionné par la voie de pénétration prépondérante et ensuite, si l'organisme dispose d'un milieu dénué de polluants radioactifs, par la décontamination différentielle des divers organes. Il s'ensuivra des doses d'irradiation différentes selon la voie de contamination et selon que l'organisme est en phase d'accumulation ou d'élimination du radionucléide. 
En raison d'une accumulation beaucoup plus importante après contamination par l'eau les animaux subissent une irradiation globale plus forte qu'après une contamination par voie alimentaire. Toutefois cette première approximation est à nuancer si nous comparons d'une part les doses d'irradiation (photons et rayonnements $\beta$ ) au centre de l'animal dues à la contamination des organes critiques externes (coquille et manteau, branchies, carapace et pièces buccales) après contamination par l'eau et d'autre part les doses d'irradiation (photons et rayonnements $\beta$ ) subies par les organes critiques internes (hépatopancréas) après contamination par la nourriture. $C e$ phénomène déjà net pour les photons est encore renforcé par l'émission de rayonnements $\beta$ car ces derniers ont un parcours plus limité. En effet la quasi-totalité des rayonnements $\beta$ résultant de la contamination des organes externes, dont la moitié se perd dans le milieu extérieur, ne parviendra pas au centre de l'animal. De plus la contamination externe, même importante pondéralement $(\mathrm{nCi} / \mathrm{g})$, est répartie sur une surface beaucoup plus grande qu'une contamination interne. Or, les organes les plus radiosensibles sont, dans la plupart des cas, internes comme les organes génitaux. Chez les Mollusques et les Crustacés les organes génitaux sont aussi souvent très près de l'hépatopancréas, organe stockeur principal. Il en résultera que, même pour des concentrations nettement plus élevées après contamination par l'eau, l'accumulation de l'argent $110 \mathrm{~m}$ par voie alimentaire risque d'avoir des conséquences nocives plus importantes.

\section{RESUME}

L'organotropisme de l'argent $110 \mathrm{~m}$ chez un Mollusque Scrobicularia plana et un Crustacé Carcinus maenas varie considérablement selon la voie de contamination: après contamination directe par l'eau ce sont les organes externes qui accumulent le plus l'argent $110 \mathrm{~m}$ (coquille et manteau de la Scrobiculaire, exosquelette et branchies du Crabe) tandis qu'après contamination par l'intermédiaire de la nourriture c'est l'hépatopancréas qui retient la quasi-totalité de l'argent radioactif. La mue des Crustacés aura une grande importance dans la décontamination de l'animal après contamination par l'eau car l'exuvie est constituée de tous les organes les plus contaminés. L'autoépuration des divers organes des animaux se fait différentiellement selon la voie de contamination. Elle est en général rapide pour les organes externes et lente pour l'hépatopancréas. Malgré une contamination importante des organes externes, ces derniers entraînent une dose d'irradiation au centre de l'animal plus faible que celle subie par l'hépatopancréas.

\section{LITTERATURE CITEE}

Amiard, J.-C., 1978a. Modalités de la contamination d'une chaîne trophique marine benthique par l'argent $110 \mathrm{~m}$. 1. Contamination de quelques organismes marins par l'intermédiaire de l'eau. Cah. Biol. mar. (Sous presse).

- 1978 b. Modalités de la contamination d'une chaîne trophique marine benthique par l'argent $110 \mathrm{~m}$. 2. Transfert du radionucléide dans la chaîne alimentaire. Cah. Biol. mar. (Sous presse). 
- 1978 c. Contribution à l'étude de l'accumulation et de la toxicité de quelques polluants stables et radioactifs chez des organismes marins: l'antimoine, l'argent, le cobalt et le strontium chez des Mollusques, des Crustacés et des Téléostéens. Thèse de Doctorat d'Etat, Université P. et M. Curie, Paris, 150 pp. (Rapport CEA-R-4928).

Beasley, T. M. \& Held, E. E., 1971. Silver-108 $\mathrm{m}$ in biota and sediments at Bikini and Eniwetok Atolls. Nature, Lond. 230, 450-451.

Drach, P., 1939. Mue et cycles d'intermue chez les Crustacés Décapodes Annls Inst. océanogr., Monaco 19, 103-391.

- \& Tchernigovtzeff, C., 1967. Sur la méthode de détermination des stades d'intermue et son application générale aux Crustacés. Vie Milieu 13 (A), 595-610.

Fitzgerald, J. J., Brownell, G. L. \& Mahoney, F. J., 1967. Mathematical theory of radiation dosimetry. Gordon \& Breach, New York, 747 pp.

Folsom, T. R., Grismore, R. \& Young, D. R., 1970. Long-lived y ray emitting nuclide silver$108 \mathrm{~m}$ found in Pacific marine organisms and used for dating. Nature, Lond. 227, 941-943.

- \& Young, D. R., 1965. Silver-110 $\mathrm{m}$ and Cobalt-60 in oceanic and coastal organisms. Nature, Lond. 206, 803-806.

Fukai, R. \& Murray, C. N., 1974. Environmental behaviour of radiocobalt and radiosilver released from ruclear power stations into aquatic systems. In: Environmental behaviour radionuclides released in the nuclear industry. IAEA, Vienna, 217-242.

Greig, R. A., 1975. Comparison of atomic absorption and neutron activation analyses for the determination of silver, chromium, and zinc in various marine organisms. Analyt. Chem. 47 (9), 1682-1684.

Grismore, R., Folsom, T. R., Hodge, V. F. \& Young, D. R., 1972. A study of the silver signature of the 1961-62 nuclear weapons testing period. Trans. N. Y. Acad. Sci. (Ser. 2) 34, 392-415.

Isackson, J. S. \& Seymour, A. H., 1968. Radiometric and elemental analyses on marine organisms from Amchitka, Alaska. BMI-171-113, 1-27.

Joffre, H. \& Pages, L., 1968. Coefficients d'atténuation massique et d'absorption massique en énergie pour les photons de $10 \mathrm{keV}$ à $10 \mathrm{MeV}$. Rapport CEA-R-3655, 1-59.

Lamotte, M., 1967. Initiation aux méthodes statistiques en biologie. Masson, Paris, 144 pp.

Lederer, C. M., Hollander, J. M. \& Perlman, I., 1967. Table of isotopes. Wiley, New-York, $592 \mathrm{pp}$.

Lefort, G., 1967. Mathématiques pour les sciences biologiques et agronomiques. Colin, Paris, $679 \mathrm{pp}$.

Legrand, J., Perolat, J. P., Lagoutine, F. \& Le Gallic, Y., 1975. Table des radionucléides. C. E. A., Laboratoire de Métrologie des Rayonnements Ionisants, Saclay, 10 pp.

Lowman, F. G., Phelps, D. K., Ting, R. Y., Martin, J. H., Swit, D. J., McLin, E. R., 1967. Marine biology program progress summary report 5, (PRNC-104), 83-89.

Pouvreau, B. \& Amiard, J.-C., 1974. Etude expérimentale de l'accumulation de l'argent $110 \mathrm{~m}$ chez divers organismes marins. Rapport CEA-R-4571, 1-21.

Preston, A., Dutton, J. W. R. \& Harvey, B. R., 1968. Detection, estimation and radiological significances of silver-110 $\mathrm{m}$ in oysters in the Irish Sea and the Blackwater Estuary. Nature, Lond. 218, 689-690.

Rockwell, T., 1956. Reactor Shielding design manual. McGraw-Hill, New York, 481 pp.

Scheidhauer, J., Ausset, R., Planet, J. \& Coulon, R., 1974. Programme de surveillance de l'environnement marin du centre de La Hague. In: Population dose evaluation and standards for man and his environment. IAEA, Vienna, 347-366.

Seymour, A. H., 1963. Radioactivity of marine organisms from Guam, Palau and the Gulf of Siam, 1958-59. In: National symposium on radioecology. Ed. by: V. Schultz \& A. W. Klement. Reinhold, New York, 1, 151-157.

Zesenko, A. Ya., 1965. The distribution of radionuclides within marine animals. In: Voprosy Gidrobiologiya. Nauka, Moscow, 174-175. 